\title{
Modeling, Evaluation and Simulation of a Supercapacitor Module for Energy Storage Application
}

\author{
A. B. Cultura II \\ Electrical Engineering Department \\ Mindanao University of Science and Technology
}

\author{
Z. M. Salameh \\ Department of Electrical and Computer Engineering \\ University of Massachusetts Lowell
}

\begin{abstract}
This paper presents the electrical and mathematical model of the supercapacitor. The equivalent mathematical model derived from electrical model was used to simulate the voltage response of the supercapacitor. The model has been implemented using Matlab software program. Simulation and experimental results of the voltage charging/discharging of the supercapacitor are compared. It was found out that the results obtained using the model is in good agreement with the experimental one. Moreover a mathematical model for the efficiency as a function of discharge time was developed and presented. The effect of charge/discharge rate on the supercapacitor's temperature is also experimentally considered. Experiments show that during charging the temperature rises more than during discharging.
\end{abstract}

Keywords-Supercapacitor;Efficiency;Energy

Storage; Temperature

\section{I.INTRODUCTION}

The high price of fossil fuels and environmental concern prompted every nation to conserve energy and pour out grants to find and conduct research on the use of alternative energy sources. The concern also grows over fossil fuel usage, in terms of global warming and resource depletion, which will result to a rapid increase in the use of renewable energy such as wind and photovoltaic. But these renewable energies require efficient and reliable energy storage [1]. Although renewable energy is free and environment friendly source of electricity, a storage element is required as an energy buffer in wind and photovoltaic systems to bridge the gap between available and required energy. The lead acid battery is generally the most popular energy storage device, because of its low cost and wide availability. However photovoltaic panels or wind are not an ideal source for battery charging as the output is unreliable and heavily dependent on weather conditions. The batteries cannot immediately response to the load requirement when sudden clouds or no wind occur. The batteries are often deep discharged, which damages the battery and shortens its useful life. It is not possible to ensure an optimum charge/discharge cycle. Undercharging of the battery leads to sulfation and stratification; both of which shorten the lifetime of the battery. Another cause of reduced battery life is gassing, which results from battery overcharging [2].

One of the latest energy storage introduced to the market today is the supercapacitor. Supercapacitors are components for energy storage, dedicated for applications where both energy and power density are needed. Even if their energy density is ten times lower than the energy density of batteries, supecapacitors offer new alternatives for applications where energy storage is needed [3]. Supercapacitor has high power density but low in terms of energy density. This supercapacitor can be used in electric or hybrid vehicles in order to provide peak power for improved acceleration and for energy recovery. It can be connected in parallel with the vehicle battery during start up of an internal combustion engine with the purpose of decreasing the size and the power of the battery, and in fuel cell vehicles in order to reduce the power and therefore the cost of the fuel cell [4]. Supercapacitor can also be used as additional energy storage for hybrid wind and photovoltaic system. It can be connected in parallel with the battery for the purpose of charging or discharging high power in a short period of time. It charges energy when it is windy or sunny and discharges when no power generated from photovoltaic or wind due to the sudden passing clouds disturbance or very low wind speed. Supercapacitors can be a good supplement for batteries in the operation of wind and PV energy storage especially during heavily variable weather conditions. Hence, it is very important to determine and understand the characteristics of the supercapacitor especially in its voltage and energy responses during charging and discharging. This paper presents the modeling and simulation of a Maxwell 48V series BMOD0140-E048 supercapacitor module for energy storage applications.

\section{SUPERCAPACITOR MODELING}

\section{A. Definition of Super Capacitor}

A supercapacitor is a high-energy version of a conventional capacitor, holding hundreds of times more energy per unit volume or mass. A supercapacitor is an electrochemical device consisting of two porous electrodes immersed in an electrolyte solution that stores charge electrostatically. The capacitance is determined by the effective-area of the plates, the separation distance of the plates and the dielectric constant of the separating medium which is the same in the conventional capacitor. A conventional capacitor gets its area from plates of a flat, conductive material while supercapacitor gets its area from a porous carbon-based electrode material. The porous structure gives it a very high effective surface area compared to a conventional plate structure. It also insures a minimal distance between the "plates". These two factors lead to a very high 
capacitance compared to a conventional electrolytic capacitor." Supercapacitors can have 100 to 1000 times the capacitance per unit volume compared to a conventional electrolytic capacitor [5].

\section{B. Modeling of the Supercapacitor}

The equivalent circuit used for conventional capacitors can also be applied to supercapacitors." Figure 1 shows the schematic circuit diagram as a representation of the first-order model for a supercapacitor given by [3].

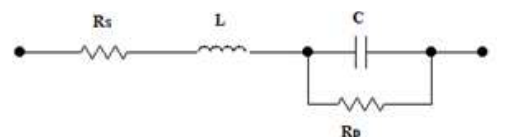
FIGURE I. THE FIRST-ORDER CIRCUIT MODEL OF A
SUPERCAPACITOR.

There are four ideal elements of the circuit given in figure 1, namely: a capacitance $C$, a series resistor $R_{S}$, a parallel resistor $R_{p}$, and a series inductor $L$. During charging and discharging, series resistance which is also called the equivalent series resistance (ESR) contributes to energy loss. Leakage current resistance which is the parallel resistance $R_{p}$ also takes energy loss due to capacitor self-discharge. In a practical capacitor $R_{p}$ is always much higher than $R_{s}$, that is why $R_{p}$ can be neglected particularly in high-power applications.

A cell of a supercapacitor can be modeled using some standard circuit components as shown in figure 1 . The elementary structure of supercapacitor, which is based on the double layer capacitor technology, consists of two activated porous carbon-based electrodes [6]. The porous structure of this material allows its surface area to approach two thousand square-meters per gram. The measured capacitance of activated carbon shows a non linear relationship with their surface area, which result primarily from the porous material used to form the electrodes that cause the resistance and capacitance to be distributed whose electrical response is equivalent to the transmission-line model shown in figure 2 which shows a more accurate illustration of the equivalent circuit of the supercapacitor [6]. Hence, supercapacitor theoretical model could be treated as a transmission line with the voltage dependent distributed capacitance.

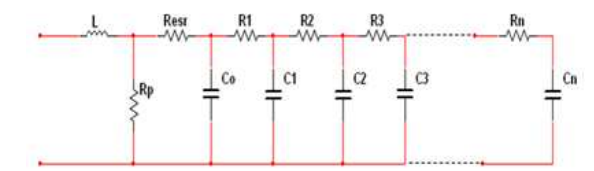

\section{FIGURE II. EXACT EQUIVALENT CIRCUIT OF THE} SUPERCAPACITOR.

However, the proposed simplified equivalent circuit of supercapacitor cell used in this study is shown in figure 3 where other RC's slow branch circuits are neglected. The classical equivalent circuit model illustrated in figure 3 is the simplest of the supercapacitor models and can adequately describe the capacitors performance in slow discharge applications (in the order of a few seconds).This model circuit is used to describe the terminal behavior of the supercapacitor.
The total capacitance of the supercapacitor cell is dependent on the voltage of the supercapacitor whose value can be express as a constant capacitor and a variable capacitor that varies linearly with the cell voltage (Vcell). Hence, the equivalent capacitance can be expressed as;

$$
C_{\text {cell }}=C_{O}+k V_{C}
$$

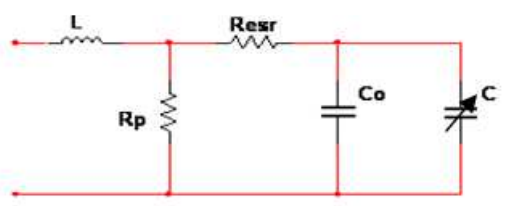

\section{FIGURE III. SIMPLIFIED EQUIVALENT CIRCUIT OF THE} SUPERCAPACITOR.

Resr is the equivalent series resistance that contributes to the energy loss during charging and discharging of a supercapacitor. $R_{p}$ is the equivalent parallel resistance that simulates energy loss due to supercapacitor self-discharge. Resistor $\mathrm{R}_{\mathrm{p}}$ is always higher than Resr and can be neglected during fast charge/discharge. While $\mathrm{L}$ is an inductor, it results primarily from the physical construction of the supercapacitor and it is usually very small. The supercapacitor used in this study comprises of 18 supercapacitor cells connected in series. Each cell has an operating voltage of 2.7 per cell, so the total terminal voltage is 48.6 volts. The overall simplified proposed electric model of the supercapacitor used in this study, neglecting the parallel resistance and the inductance, is presented in figure 4 .

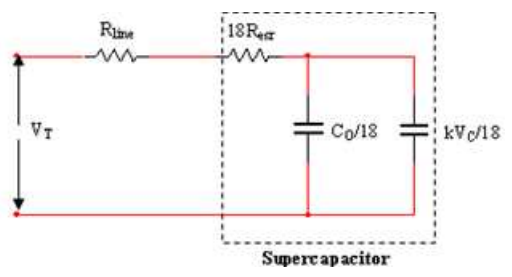

FIGURE IV. DERIVED EQUIVALENT CIRCUIT OF A MAXWELL 48V SERIES BMOD0140-E048 SUPERCAPACITOR.

The total capacitance for the 18 cells in series is;

$$
\begin{gathered}
C_{\text {total }}=\frac{1}{\frac{1}{C_{\text {cell } 1}}+\frac{1}{C_{\text {cell } 2}}+\ldots . .+\frac{1}{C_{\text {cell1 } 18}}} \\
C_{\text {total }}=\frac{1}{18} C_{\text {cell }}=\frac{1}{18}\left(C_{O}+k V_{C}\right)
\end{gathered}
$$

And the total equivalent series resistance is;

$$
R_{E S R}=18\left(R_{e s r}\right)
$$

Kirchhoff's voltage law says the total voltages in a close loop must be zero. So applying this law in figure 4 when charging a supercapacitor will have this equation: 


$$
\left(R_{\text {line }}+R_{E S R}\right) i+\frac{1}{C_{\text {total }}} \int i d t=V_{T}
$$

This equation can be expressed in differential equation in terms of charge (q) as,

$$
\left(R_{\text {line }}+R_{E S R}\right) \frac{d q}{d t}+\frac{1}{C_{\text {total }}} q=V_{T}
$$

while: $\mathrm{q}=\mathrm{CV}$

Thus, it can be expressed in terms of the voltage of the supercapacitor $\left(\mathrm{V}_{\mathrm{C}}\right)$;

$$
\left(R_{\text {line }}+R_{e s r}\right)\left(C_{O}+2 k V_{C}\right) \frac{d v_{C}(t)}{d t}+v_{C}(t)=V_{T}
$$

or

$$
\frac{d v_{C}}{d t}=\frac{V_{T}-v_{C}}{\left(R_{\text {line }}+R_{\text {esr }}\right)\left(C_{O}+2 k v_{C}\right)}
$$

Solving for the voltage across the internal resistance;

$$
R_{E S R} i(t)+\frac{1}{C_{\text {total }}} \int i(t) d t=v_{t}
$$

At the instant of switching, $\mathrm{t}=0^{+}$;

$$
R_{E S R} i(t)-\frac{1}{C_{\text {total }}} \int i(t) d t=V_{o}
$$

Multiply by $\mathrm{C}_{\text {total }}$ and take a derivative;

$$
C_{\text {total }} R_{E S R} \frac{d i(t)}{d t}-i(t)=0
$$

Multiply by $\mathrm{R}_{\mathrm{ESR}}$ and note that $\mathrm{v}_{\mathrm{r}}(\mathrm{t})=\mathrm{R} \mathrm{i}(\mathrm{t})$

$$
R_{E S R} C_{\text {total }} \frac{d v_{r}(t)}{d t}-v_{r}(t)=0
$$

And the solution of equation 7 is,

$$
v_{r}(t)=k e^{\frac{1}{R_{E S R} C_{\text {total }}} t}
$$

Hence, the voltage across the terminal of the supercapacitor is,

$$
v_{t}(t)=v_{r}(t)+v_{c}(t)
$$

and for discharging a supercapacitor, the equation is;

$$
\frac{d v_{C}}{d t}=\frac{-\left(v_{C}\right)}{\left(R_{\text {line }}+R_{\text {esr }}\right)\left(C_{O}+2 k v_{C}\right)}
$$

and the terminal voltage of the supercapacitor in discharging is,

$$
v_{t}(t)=v_{c}(t)-v_{r}(t)(11)
$$

\section{Modeling for Energy Efficiency of the Supercapacitor}

As it was mentioned in section 2, one of the main parameters of supercapacitor is its series resistance. Internal losses are due to this resistor. Many manufacturers succeeded in reducing the value of this resistor but still its value induces an energy efficiency lower than unity which will cause the reduction of available power. The energy efficiency of the supercapacitor has to be considered especially in sizing a supercapacitive bank. The way supercapacitors are charged or discharged will influence their performances [7]. So it is very important to evaluate the energy efficiency of supercapacitors at different level of operating voltages before it will be used in a certain application. This will be the bases for developing power electronics that will control the current and voltage for loading and unloading which will optimize the performances of supercapacitors.

The energy efficiency is greatly affected by the values of the internal series resistance $(\mathrm{R})$ and capacitance $(\mathrm{C})$. A relationship between efficiency and discharging time has been established from the experimental results [8].

$$
\eta_{e f f}=e^{-\frac{2 R_{E S R} C_{T O T A L}}{t_{d c h}}}
$$

The discharging time can be express in terms of voltage discharge ratio which can be derived from the equivalent circuit of the supercapacitor connected to load as shown in figure 5 .

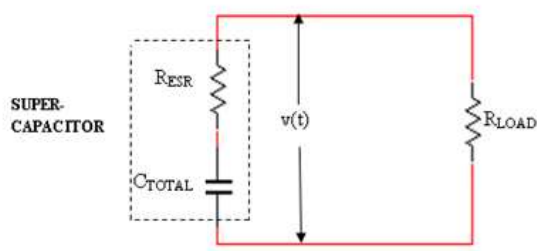

\section{FIGURE V. EQUIVALENT CIRCUIT OF DISCHARGING THE} SUPERCAPACITOR.

The voltage $\mathrm{v}(\mathrm{t})$ can be determined from the equation of a parallel RC circuit, subject to the initial condition that $\mathrm{v}(0)=$ $\mathrm{V}_{\mathrm{o}}$, [14], the $\mathrm{v}_{\mathrm{C}}(\mathrm{t})$ of the circuit above can be calculated and expressed as

$$
v_{C}(t)=V_{o} e^{-t / R_{T} C_{\text {total }}}
$$

and $v(t)=v_{C}(t)+v_{R_{E S R}}(t)$ 
so that,

$$
v(t)=V_{o}\left(1+\frac{R_{E S R}}{R_{T}}\right) e^{-t / R_{T} C_{\text {total }}}
$$

Voltage discharge ratio can be express as;

$$
d=\frac{v(t)}{V_{o}}=\left(1+\frac{R_{E S R}}{R_{T}}\right) e^{-t / R_{T_{t}} C_{\text {total }}}
$$

And solving for the discharge time from equation 15;

$$
t_{d t}=-\left(R_{E S R}+R_{\text {Load }}\right) C_{\text {total }} \ln \left[\frac{\left(R_{E S R}+R_{\text {Load }}\right) d}{2 R_{E S R}+R_{\text {Load }}}\right]
$$

Where $R_{\text {Load }}$ is a function of voltage (v) since the current (i) during discharging was set to be constant.

\section{THE SERIES BMOD0140 SUPERCAPACITOR}

The supercapacitor used in this experiment is the series BMOD0140-E048 as shown in figure 6 which is one of the supercapacitor's products manufactured by Maxwell Technologies Company. The module comprised of eighteen supercapacitor cells with an operating voltages of 2.7 per cell. The output terminal voltage is 48.6 volts. This module has an equivalent capacitance of 140 farad. The modules include bus bar connections and cell balancing circuitry. It was mentioned in the user's manual that the module-to-module cable is required for balancing series connected modules. The module balancing prevents any voltage offset accumulation, as modules are connected in series. Table 1 shows the manufacturer's specification for the BMOD0140-E048 supercapacitor.

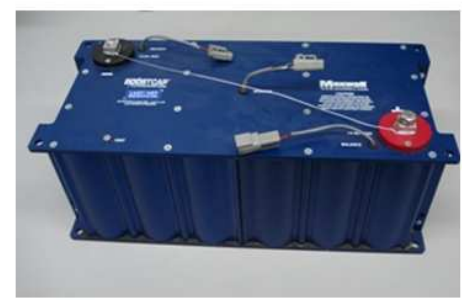

FIGURE VI. THE 48V SERIES BMOD0140-E048 SUPER CAPACITOR.
TABLE I .SPECIFICATION BMOD0140-E048 SUPERCAPACITOR.

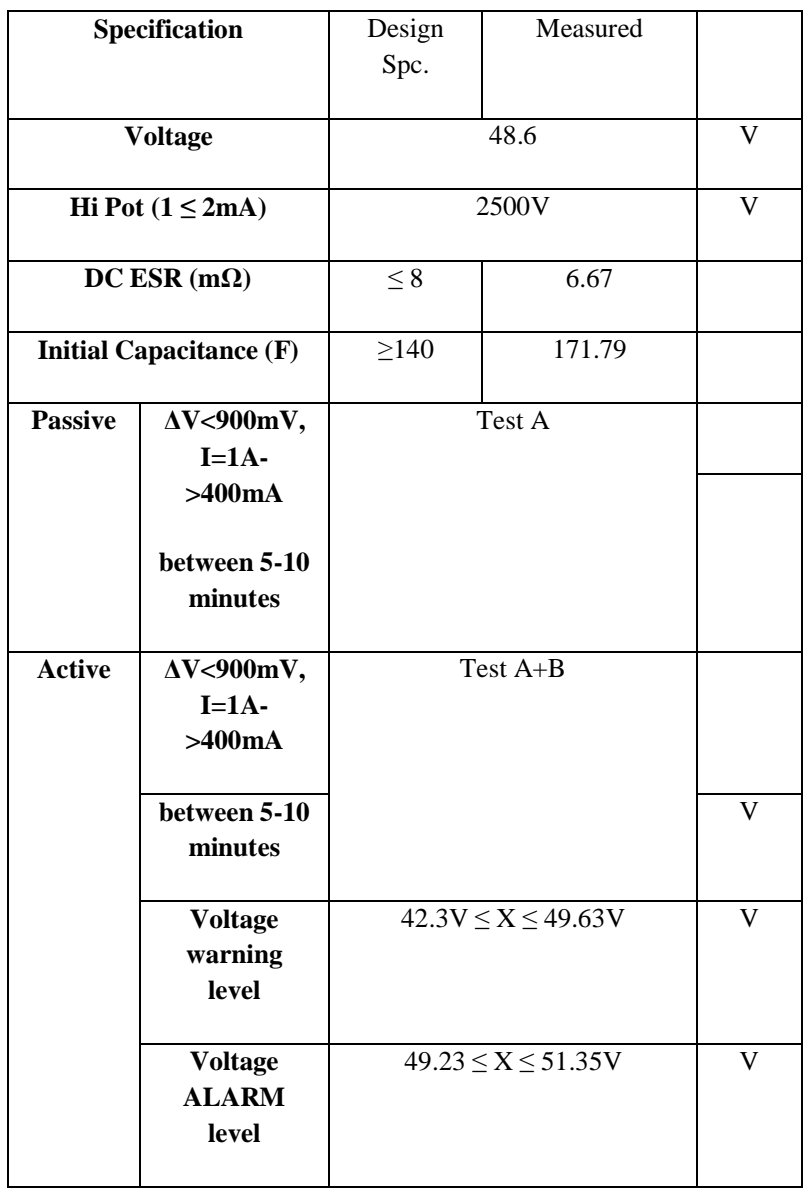

The discharge profile of a supercapacitor is shown in figure 7 as specified in the manufacturer's application note. There are two components of supercapacitor voltage profile; a capacitive component and a resistive component. The capacitive component represents the voltage change due to the change in energy within the supercapacitor and the resistive component represents the voltage change due to the equivalent series resistance (ESR) of the supercapacitor.

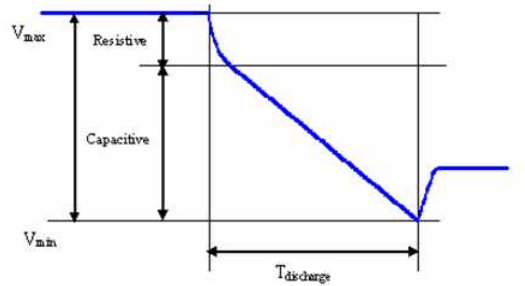

FIGURE VII. DISCHARGE PROFILE OF A SUPERCAPACITOR.

Capacitive is the voltage drop due to discharge which is expressed as

$$
d V=i \cdot \frac{d t}{C}
$$


The resistive component is given by the equation;

$$
V=i \cdot R
$$

Equation 19 is the sum of equations 17 and 18 which is the total voltage change when charging or discharging the supercapacitor.

$$
d V=i \cdot \frac{d t}{C}+i \cdot R
$$

\section{SUPERCAPACITOR'S EVALUATION}

The authors designed and fabricated an evaluation station for research on supercapacitor as shown in figure 8 and figure 9. This station is used to test, control and monitor the charging and discharging of supercapacitor. Figure 8 is the block diagram of the supercapacitor evaluation station installed at the renewable energy laboratory of University of Massachusetts (Umass) Lowell.

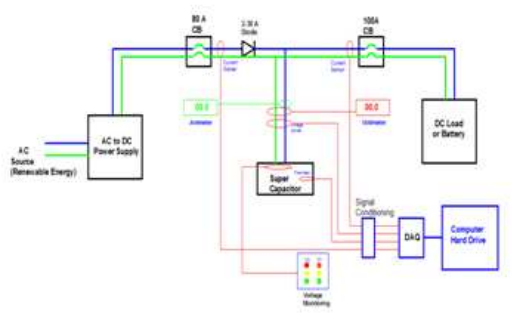

FIGURE VIII. SUPERCAPACITOR TESTER BLOCK DIAGRAM.

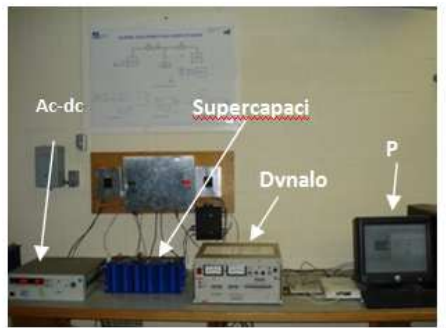

\section{FIGURE IX. . SUPERCAPACITOR EVALUATION}

The system consists of ac-dc power supply, circuit protection, diodes, dc load, sensors, data acquisition and personal computer. The Ac-dc power supply is used to charge the supercapacitor by a constant power/current charging or it can directly be charge from the $48 \mathrm{~V}$ dc terminal of the $10.56 \mathrm{~kW}$ photovoltaic system. Charge current flows through the $80 \mathrm{~A}$ circuit breaker and passing through $60 \mathrm{~A}$ diode before going to the supercapacitor. The purpose of the diode is to prevent a surge current flowing from supercapacitor back to the power supply. The dc dynaload is used to discharge energy from the supercapacitor. Discharge current flows from supercapacitor passing through a $100 \mathrm{~A}$ circuit protection and going to the dc dynaload. Sensors (for current, voltage and temperature) were used to send a signal input to conditioning circuit before sending it to the data acquisition and save the data in the hard drive of the computer. Signal conditioning circuits for current, voltage and temperature were designed for the data acquisition to accept input signal. During the evaluation, data will be collected through the NI USB DAQ and will be automatically saved in the hard drive of the computer using a Lab View software program.

A simple current charging with standard power supply was being used in charging a supercapacitor. The current limit was being set to 20 amperes and the voltage limit was being set to the maximum required voltage. Figure 10 shows the curves of charging a supercapacitor at 20 amperes constant charging current, wherein it took 405 seconds to fully charge the supercapacitor at 51.8 volts. In discharging, the supercapacitor is connected to a dc dynaload. This load was being set at different level of constant current. After every fully charge of a supercapacitor, it was discharged at different level of constant current at 20,40, and 60 Amperes. Figure 11 shows the curves at different load currents. The total times of fully discharging a supercapacitor were 370, 187 and 121 seconds at load current of 20,40 and 60 amperes respectively. Data from the experiment shows that the capacitance of the supercapacitor cell varies linearly with the cell voltage $\left(\mathrm{V}_{\text {cell }}\right)$ as shown in figure 12 .

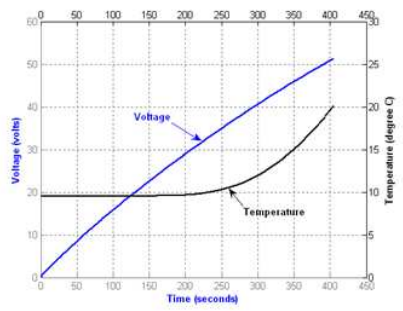

FIGURE X. CHARGE PROFILE OF SUPERCAPACITOR AT 20A.

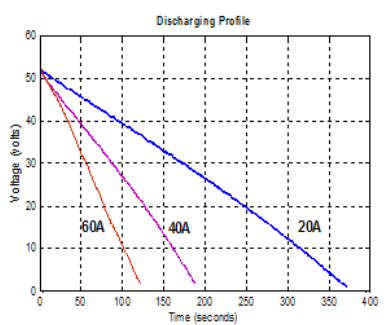

FIGURE XI. DISCHARGE PROFILE OF SUPER CAPACITOR.

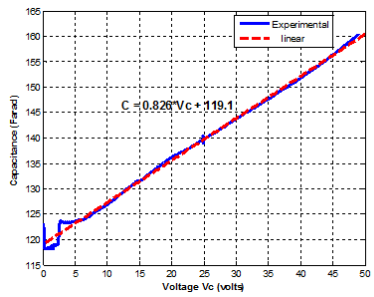

FIGURE XII. VARIATION OF CAPACITANCE OF THE SUPERCAPACITOR WITH RESPECT TO CELL VOLTAGE. 


\section{SUPERCAPACITOR's EVALUATION}

\section{A. Charge and Discharge Voltage of the Supercapacitor}

The mathematical model derived from the proposed electrical model of the supercapacitor is implemented using the Matlab software program. Figure 13 shows the charge profile of the supercacitor at supply surrent of 20 Amperes where dashed and solid curve are represented as simulation and experimental results respectively. It is clear that the results obtained using the model is in good agreement with the experimental one. Hence, the proposed electric model is validated and can be used in designing a voltage controller and in sizing a supercapacitor for storage application.

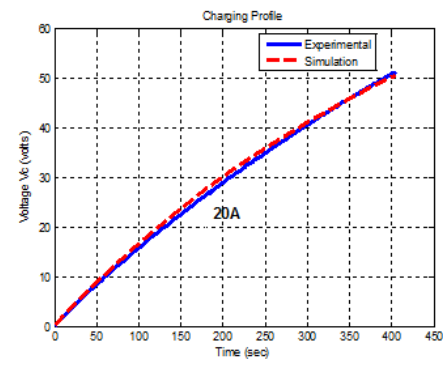

FIGURE XIII. CHARGE PROFILE OF THE SUPERCAPACITOR.

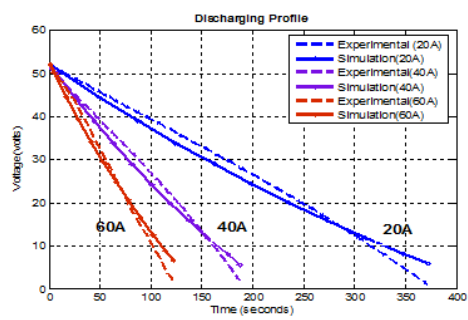

FIGURE XIV. .DISHARGE PROFILE OF THE SUPERCAPACITOR .

Figure 14 shows the discharge profile of the supercacitor at load currents of 20,40 and 60 Amperes where dotted and solid curves are simulation and experimental results respectively. It shows that simulation and experimental results are in good agreement whose minor difference attributed to the effect of the approximated electrical model. Although the derived equivalent model is not a perfect model, it gives a good approximation of the supercapacitor characteristics when compared to a real device in slow discharge applications.

Using this model, the authors can estimate and simulate of how much time the supercapacitor can be fully charge at high level of supply current. To adjust the supply current to a certain $\mathrm{k}$ factor, the left side of equation (5) must be divided by a $\mathrm{k}$ factor. Results in the simulation show that total times of fully charging a supercapacitor at rated voltage were 405, 202, and 135 seconds at supply current of 20,40, and 60 Amperes respectively as shown in figure 15 .

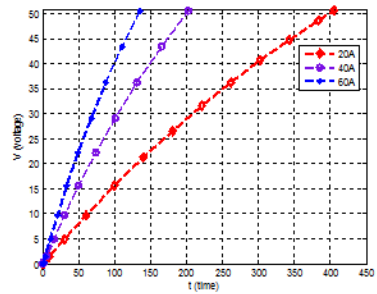

FIGURE XV. SIMULATED CHARGE PROFILE OF THE SUPERCAPACITOR AT 20,40 AND 60 AMPERES .

\section{B. Experimental Results of the Effect of Charge/Discharge on Supercapacitor'S Temperature}

Temperature of the supercapacitor during operation was being monitored since it is very important to determine how the temperature behaves when the supercapacitor is operated at different level of operating current so that it will be protected from damage. Modeling of the temperature of the supercapacitor is not being considered in this study but results of the temperature variations during evaluation are being presented. When a supercapacitor was charged for the first time at 20 amperes supply current, the temperature was initially at a constant of $9.8^{\circ} \mathrm{C}$ for approximately 150 seconds and it started to increase exponentially until it was fully charge. Figure 16 shows the curves of temperature when the supercapacitor was charged at different initial temperature at 20 amperes charging. In all cases the supercapacitor temperature was initially constant and then it increases exponentially after 150 seconds of charging.

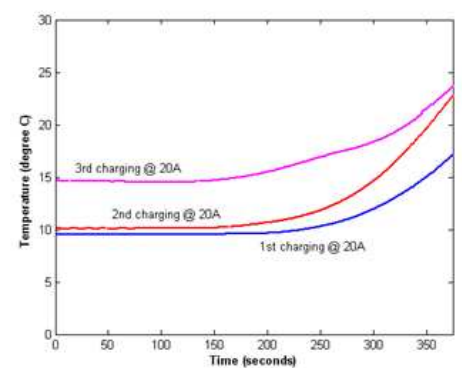

FIGURE XVI. TEMPERATURE VARIATIONS OF A SUPERCAPACITOR CHARGING.

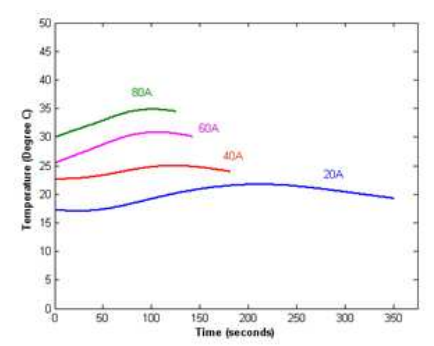

FIGURE XVII. TEMPERATURE VARIATIONS OF A SUPERCAPACITOR DURING DURING DISCHARGING.

Figure 17 shows the curves of the temperatures of the supercapacitor as a function of the discharge time for different constant load currents. The temperature varies sinusoidally as a function of discharge time. But temperature range increases 
as load current increases. When the load current was $20 \mathrm{~A}$, the temperature operates at the range of $17.0^{\circ} \mathrm{C}$ to $21.67^{\circ} \mathrm{C}$, and when it was discharged at 80 amperes, the temperature range increased and became $30^{\circ} \mathrm{C}$ to $34.8^{\circ} \mathrm{C}$.

\section{DISCUSSIONS AND CONCLUSIONS}

This paper presents the electrical and mathematical modeling of the supercapacitor. The simulation of the charge/discharge voltage characteristics of a model supercapacitors are carried out using the mathematical model derived from the electrical model. The charge and discharge voltage behavior of the supercapacitor is simulated and experimented. It is concluded that the model is well capable of simulating the voltage behavior and can predict the time of charge/discharge voltage of the supercapacitor. Although the derived equivalent model is not a perfect model, it gives a good approximation of the supercapacitor characteristics when compared to a real device in slow discharge applications.

The energy efficiency of the supercapacitor was also modeled using the derived formula. This model was verified experimentally [8]. The experimental results show that the energy efficiency of the supercapacitor increases as a function of the discharge time. Since the discharge time is a function of the discharge current, so the higher the discharge current the lower the discharge time and the energy efficiency. The temperature effect of the supercapacitor was also experimented. It was observed that during charging the temperature stays constant for a while and then it increases exponentially. And during discharging it varies in a sinusoidal form. Experiments show that during charging the temperature rises more than during discharging.

Moreover, further study and analysis will be done when the supercapacitor will be connected to the existing wind/PV hybrid system as planned. In fact, supercapacitor is a good choice for peak power applications because of its high power density. If it is use in a hybrid system, the supercapacitor works with the battery to supply power to the load when the solar panel or wind turbine is not producing enough power [19]. In this situation the battery supplies constant low power to the load while the supercapacitor supplies the peak power requirements. This approach will result in an extension of the battery life and a reduction in the size of the battery pack as expected.

\section{REFERENCES}

[1] A. K. Shukla*, S. Sampath and K. Vijayamohanan, Electrochemical supercapacitors: Energy storage beyond batteries, Current Science, VOL. 79, No.12, 25 December 2000

[2] M.E. Glavin and W.G. Hurley, Ultracapacitor/Battery Hybrid for Solar Energy Storage, National University of Ireland Galway, Ireland, IEEE Trans., pp791-795 April 7, 2009

[3] Philippe Barrade, Alfred Rufer, Current Capability and Power Density of Supercacitors: Considerations on Energy Efficiency, LEI, STI-ISE and EPEL, CH-1015 Lausanne, Switzerland EPE 2003

[4] F.Rafik, H.Gualous, R. Gallay, M.Karmous, A.Berthon, Contribution to the Sizing of Supercapacitors and their Applications, Ecole d'ingénieurs Arc, CH-2400 LE LOCLE, Switzerland L2ES-UTBM-Univ.Franche comté, Rue T.MIEG, F90010 BELFORT, France, MAXWELL Technologies, CH-1728 Rossens, Switzerland
[5] Philip P. Barker, Ultracapacitors for Use in Power Quality and Distributed Resource Applications, EPRI PEAC Corporation, 942 Corridor Part Blvd., Knoxville, TN 37932

[6] Youngho Kim, Director of Product Development, Ultracapacitor Technology Power Electronic Circuits, NESSCAP Co. Ltd., Korea

[7] P. Barrade, Energy Storage and Applications with Supercapacitors, Laboratorie d'Electronique Industrielle, LEI STI-ISE, Ecole Polytechnic Fererale de Lausanne, EPFL CH 1015 Lausanne (Switzerland)

[8] Ambrosio B. Cultura, Ziyad M. Salameh, Performance Evaluation of a Supercapacitor Module for Energy Storage Application, Presented Power and Energy Society General Meeting - Conversion and Delivery of Electrical Energy in the 21st Century, 2008 IEEE

[9] F. Rafik, et. al, Super Capacitors Characterization For Hybrid Vehicle Applications, Haute Ecole Arc Ingenierie, CH-2400 LE LOCLE, Switzerland L2ES-UTBM-Univ. Franche comte, Tue T. MIEG, F90010 BELFORT, France MAXWELL Technologies, CH-1728 Rossens, Switzerland

[10] Richard E. Smith, Ultracapacitor System Design; Optimizing Hybrid Electric Vehicles with Fuel Cell Power, Sr. Vice President of Strategic Business Development, Maxwell Technologies

[11] Randy B. Wright, David K. Jamison, and Jeffrey R. Belt and Raymond A. Sutula, Performance Testing Of Selected Commercial Ultracapacitors Idaho National Engineering and Environmental Laboratory P.O. Box 1625, Idaho Falls, ID 83415-3830 and US Department of Energy 1000 Independence Avenue, S. W. Washington, DC 20858

[12] Abhishek Drolia, Philip Jose, Ned Mohan, An Approach to Connect Ultracapacitor to Fuel Cell Powered Electric Vehicle and Emulating Fuel Cell Electrical Characteristics using Switched Mode Converter, Department of Electrical Engineering, University of Minesota, IEEE Trans., pp7803-7906, 2003

[13] D. Riu, N. Retiere, D. Linzen, Half-order Modelling of Supercapacitors, Laboratoire d' Electrotechnique de Grenoble and Institute for Power Electronics, LEG/ENSIEG-Domaine Universitaire and Electrical Devices, University of Technology-Jaegerstr, IEEE Trans., pp78038386-5, 2004

[14] Prof. Alfred Ruffer, Power-Electronic Interface for a SupercapacitorBased Energy-Storage Substation in DC-Transportation Networks, Laboratoire d'Electronique Industrielle, LEI Institut des Sciences de l'Energie, STI-ISE, EPE 2003-Toulouse, ISBN:90-75815-07-7

[15] William H. Hayt, Jr, Jack E. Kemmerly, Engineering Circuit Analysis, Fifth Edition, McGraw-Hill, 1993, pp.157-158.

[16] Application Note Maxwell Technologies Document 1008981 Rev 1, Charging of Ultracapacitors

[17] Maxwell Technologies User's Manual, BOOSTCAP Energy Storage Module 16 and 48 volts series

[18] Application Note Maxwell Technologies Document 1007239 Rev 1, Representative Test Procedure for Customer Evaluations

[19] Donghwa Shina, Younghyun Kima, Yanzhi Wang, Naehyuck Changa, Massoud Pedramb, Constant-current regulator-based batterysupercapacitor hybrid architecture for high-rate pulsed load applications, Elsevier Journal of Power Sources 205 (2012) 516-524 